\title{
Primary Study of Novel Method for Determination of Saponin in Ziziphi Spinosae Semen (Suan-Zao-Ren) by Digitally Scanning the Photo of the HPTLC Image without Configuration of TLC Scanner Facility
}

\author{
Longgang Guo1, Shuai Sun ${ }^{2}$, He Li ${ }^{1,3}$, Ruiyin Wang1, Xinjun $\mathrm{Xu}^{4}{ }^{*}$, Peishan Xie ${ }^{{ }^{*}}$ \\ ${ }^{1}$ Guangdong UNION Biochemical Development Co. Ltd., Guangzhou, China \\ ${ }^{2}$ Guangdong ImVin Pharmaceutical Co. Ltd., Guangzhou, China \\ ${ }^{3}$ Livzon Group Pharmaceutical Factory, Zhuhai, China \\ ${ }^{4}$ School of Pharmaceutical Sciences, Sun Yat-sen University, Guangzhou, China \\ Email: ${ }^{*}$ xxj2702@sina.com, ${ }^{*}$ psxie163@163.com, ${ }^{*}$ xps340112@gmail.com
}

Received 24 August 2014; revised 11 October 2014; accepted 28 October 2014

Copyright (C) 2014 by authors and Scientific Research Publishing Inc.

This work is licensed under the Creative Commons Attribution International License (CC BY).

http://creativecommons.org/licenses/by/4.0/

(c) (†) Open Access

\begin{abstract}
Conventional quantity determination of Chinese herbal medicines by High-Performance ThinLayer Chromatography (HPTLC) must be carried out on a TLC scanner for measuring the marker component on the HPTLC plate. Generally the HPTLC image of the herbal drug always takes the photograph as the permanent visual document. Considering the photo of the HPTLC image preserving all the visual quality-related parameters, there might be an alternative cost/effective approach worth studying. It would be presumably applicable to use the exclusively developed software as a virtual scanner instead of the TLC Scanner instrument to do digital scanning, integrating and calculating target components in the photo of the HPTLC image. It means digitally scanning the photo of the HPTLC image solely with software does the same thing as the instrumental scanner does. Based on this protocol, we primarily conducted the assay of the contents of the major saponins, jujuboside a and jujuboside b, in Ziziphi Spinosae Semen (Suan-Zao-Ren). Using home-developed software "digiscan" for acquiring the raw data and integrating the peaks area of the target components with "Origin Pro", the contents of jujuboside a and jujuboside b can then be calculated respectively. The primary investigated experiment results were well compatible with official HPLC-
\end{abstract}

${ }^{*}$ Corresponding authors.

How to cite this paper: Guo, L.G., Sun, S., Li, H., Wang, R.Y., Xu, X.J. and Xie, P.S. (2014) Primary Study of Novel Method for Determination of Saponin in Ziziphi Spinosae Semen (Suan-Zao-Ren) by Digitally Scanning the Photo of the HPTLC Image without Configuration of TLC Scanner Facility. Chinese Medicine, 5, 211-217. http://dx.doi.org/10.4236/cm.2014.54025 
ELSD method in Chinese Pharmacopoeia. The methodology validation and comparative analysis with HPLC confirmed that the unprecedented method is acceptable. The advantage of the recommended approach is far more simple, flexible and cost/effective than the conventional method, and it can be done at anytime and anywhere once only if the high quality HPTLC image photo is available. There would be a bright perspective for application to other herbal medicines. And the one-stop software for merging digital scanning, integration and calculation together is in progress.

\section{Keywords}

HPTLC Image Photo, Digital-Determination, Ziziphi Spinosae Semen (Suan-Zao-Ren), Jujuboside $a$ and $b$

\section{Introduction}

One of the common-used Chinese herbal drugs, Spine Date Seed, Chinese name: Suan-Zao-Ren (abbr. SZR) is the dried ripe seed of Ziziphus jujube Mill. var. spinosa (Bunge) Hu et H.F. Chou (Family Rhamnaceae). Its acknowledged bioactive ingredients are saponins, flavonoids, polysaccharides, fatty acids [1]-[5]; amongst those, the major bioactive saponins-jujuboside a, jujuboside $b$ and flavonoid spinosin are the chemical markers for identification and assay stipulated in Chinese Pharmacopoeia [6].

Generally determining the content of the selected marker component in the herbal drugs by means of chromatographic analysis such as HPLC, GC and HPTLC is the current basic requirement for herbal quality control. Amongst the chromatographic techniques, the off-line HPTLC technique is more convenient, economical, and flexible than others. In particular, the chromatogram pattern provided by HPTLC is a colorful picture-like image; all the images of the tested samples and the reference substances express on the same plate in parallel. The visual specific chemical composition functions effectively as either identification or quantitative analysis. That means multiple samples' qualitative (fingerprinting) and quantitative (marker quantification) testing in one-run on the same HPTLC plate is attractive for herbal medicines. The HPTLC quantitative analysis for herbal drugs' bioactive markers is conventionally carried out with expensive professional TLC scanner. The HPTLC image is considered as the permanent visual data bank, in which all of the parameters of relevant quality of the herbals are preserved. It can be presumed that the photo of the HPTLC image can play the role not only for fingerprinting identification as shown in the TLC Atlas [7] but also possibly for quantitative determination by digital scanning via software as a "virtual scanner" doing the same thing as the conventional determination of the HPTLC plate with the TLC scanner instrument. Based on such a protocol, we determined the jujuboside a and jujuboside b in Spine Date Seed (Suan-Zao-Ren, abbr. "SZR") on the HPTLC image photo with external standard method as pilot experiment in order to confirm the feasibility of the "digiscan" protocol.

\section{Methods}

\subsection{Materials, Reagents}

Ziziphi Spinosae Semen (SZR; Wild jujube seeds), purchased from the whole sale herbal drug markets in Hebei province and Guangzhou city in China; the defatted extractive of SZR (prepared in our lab; jujuboside a and jujuboside b, the purity: $>98 \%$ via calibration by external standard method); n-butyl alcohol; methanol; ethanol; sulphuric acid; vanillin (analytical grades). HPTLC silica-gel plate (Merck, Germany).

\subsection{Instrument and Software}

Sample application device: Linomat 5; HPTLC development twin-trough chamber; HPTLC reagent dipping device; TLC visualizer (all from CAMAG, Mutenz, Switzerland) and/or digital single-lens reflex camera; "digiscan” software (home-developed by Franky Du) and Origin Pro 75 (Figure 1).

\subsection{SZR Sample Solution}

Add $100 \mathrm{~mL}$ of petroleum ether (b.p. $60^{\circ} \mathrm{C}$ ) to about 4.0 g of the powdered sample, sonicated at $30^{\circ} \mathrm{C}$ for 60 

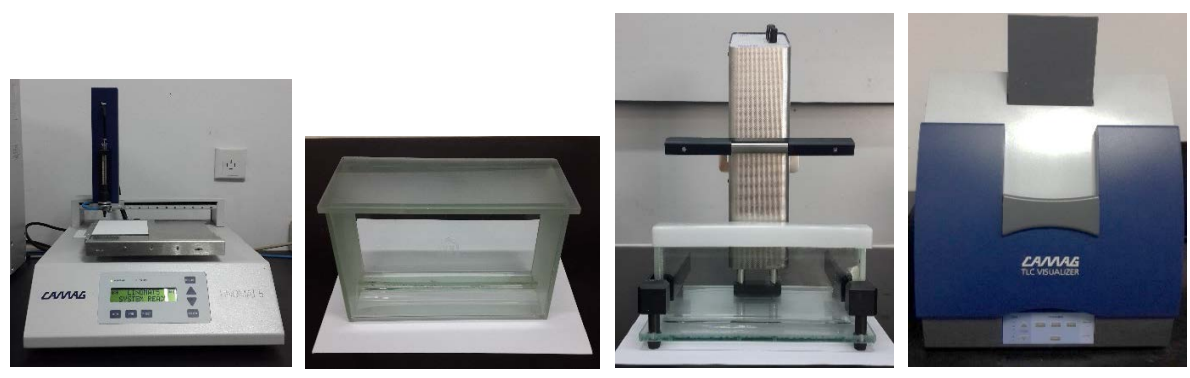

Figure 1. HPTLC facilities: Linomat 5, twin-trough chamber, reagent dipping device, CAMAG TLC Visualizer (CAMAG, Switzerland).

min, filter, discard the petroleum ether; evaporate the remnant solvent in the drug powder by flowing air, reflex extract the residue with methanol for 60 minutes, filter, evaporate the filtrate on water bath to dryness, dissolve the extract substance with $2.0 \mathrm{~mL}$ of methanol, the methanol solution ready for use.

\subsection{Defatted ESZR (ESZR) Solution}

Add $20 \mathrm{~mL}$ of methanol to $0.1 \mathrm{~g}$ defatted ESZR, sonicated for $15 \mathrm{~min}$, filter, evaporate the methanol solution to dryness, re-dissolve the residue to $1 \mathrm{~mL}$ of methanol, and filter via $0.22 \mu \mathrm{L}$ filter-membrane, the filtrate ready for use.

\subsection{Chemical Reference Substance (CRS) Solution}

Dissolve about $0.5 \mathrm{mg}$ each of jujuboside a and jujuboside b in $0.5 \mathrm{~mL}$ of methanol respectively.

\subsection{Sample Application}

Apply appropriate amount ( $\mu \mathrm{L}$ level, see Table 1 ) of the sample solution and CRS solution on HPTLC silica gel-60 plate $(20 \times 10 \mathrm{~cm}$; Merck; Lot: OB678146) by means of Linomat 5 (CAMAG). Band width: $8 \mathrm{~mm}$, interval between band to band: $4 \mathrm{~mm}$. The sample-loaded plate was kept in a vacuum desiccator $\left(\mathrm{P}_{2} \mathrm{O}_{5}\right.$ anhydrous $)$ for $1 \mathrm{~h}$ before development.

\subsection{Solvent System (Mobile Phase) and Development [6]}

Aqueous-saturated n-butyl alcohol (cf. Chinese Pharmacopoeia [6]); ascending develop to $8 \mathrm{~cm}$. T: $20^{\circ} \mathrm{C}$; $\mathrm{RH}$ : $40 \%-50 \%$.

\subsection{Visualization}

1) Dry the developed plate with flowing air to remove the remnant solvent and put it in a vacuum dessicator over $\mathrm{P}_{2} \mathrm{O}_{5}$ for 3 hours; 2) put the developed dry plate in the reagent dipping device in which $1 \%$ of vanillin/70\% of sulphuric acid ethanol solution contained, the dipping time adjusted to 1 second; 3 ) take out the plate from the chamber, wipe out the residual reagent on the back of the plate as quickly as possible; the azure color of the two saponins are clearly visible. Caution: a crucial environment condition in lower relative humidity $(\mathrm{RH}<50 \%)$ and lower temperature $\left(\mathrm{T}<20^{\circ} \mathrm{C}\right)$ during the visualization period must be kept.

\subsection{The HPTLC Image Photograph Preparation}

1) Take the visualized HPTLC plate in the TLC visualizer (CAMAG) as quickly as possible. The parameters in this case were: Exposure: Auto; Exposure time: 60.95 ms; Gain: 1.00; Digital level: 85\%; Accentuation: 0.80; Color saturation: 1.30; Contrast: 1.0; Brightness: 0.00; White balance: Red gain: 1.39; Green gain: 1.00; Blue gain: 2.34. The alternative approach is by using a digital single-lens reflex camera to take the visible color image from the visualized plate and save the picture in jpg format; 2) the "modification" of the image photo was often necessary because some "noise" on the background caused by the remnant reagent and some unexpected contamination on the surface or the bright or the contrast too weak. The parameters adjusted by Photoshop ${ }^{\circledR}$ in this 
Table 1. Recovery data.

\begin{tabular}{|c|c|c|c|c|c|c|c|c|}
\hline \multirow{2}{*}{$\begin{array}{l}\text { Target } \\
\text { amount }\end{array}$} & \multicolumn{4}{|c|}{ 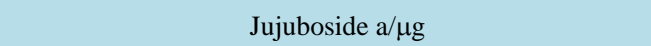 } & \multicolumn{4}{|c|}{ Jujuboside b/ug } \\
\hline & $\begin{array}{c}\text { Before } \\
\text { spiked ERS }\end{array}$ & $\begin{array}{c}\text { After } \\
\text { spiked (total) }\end{array}$ & $\begin{array}{l}\text { Spiked } \\
\text { amount }\end{array}$ & $\begin{array}{c}\text { Recovery } \\
\text { (\%) }\end{array}$ & $\begin{array}{c}\text { Before } \\
\text { spiked ERS }\end{array}$ & $\begin{array}{c}\text { After } \\
\text { spiked (total) }\end{array}$ & $\begin{array}{l}\text { Spiked } \\
\text { amount }\end{array}$ & Recovery (\%) \\
\hline $1 \mathrm{st}$ & 0.882 & 1.594 & 0.696 & 102.43 & 0.647 & 1.140 & 0.441 & 111.88 \\
\hline 2nd & 0.886 & 1.589 & 0.696 & 101.17 & 0.653 & 1.1413 & 0.441 & 110.75 \\
\hline 3rd & 0.886 & 1.593 & 0.696 & 101.70 & 0.640 & 1.1297 & 0.441 & 111.03 \\
\hline Average & \multicolumn{4}{|c|}{101.8} & \multicolumn{4}{|c|}{111.2} \\
\hline RSD & \multicolumn{4}{|c|}{0.63} & \multicolumn{4}{|c|}{0.59} \\
\hline
\end{tabular}

case were: surface blur (radius 4 pixel; threshold 100 gradation; luminance (luminance 45, contrast 18), smart sharpen (100\%, radius 10 pixel); background replace color (luminance +33$)$. The modification aimed at getting the clean and clear picture meanwhile keeping the image as what the naked-eye sighted as possible. The azure color of jujuboside a and jujuboside $b$ bands on the greenish-yellow background of the HPTLC image photo observed (Figure 2 and Figure 3).

\subsection{Digital Scanning and Data Processing}

1) Digital scanning the HPTLC image photo of $S Z R$ by self-developed software "digiscan", save the scanning raw data; 2) input the raw data to software "Origin Pro" for integration and getting the scanning profile; 3) output the integrated data into MS Excel for calculation. The facilities for HPTLC experiment refer to Figure 1.

\section{Methodology Validation}

\subsection{Linearity}

The equation of the linearity of jujuboside a: $y=76820 x-14171, R^{2}=0.9993$; jujuboside $b: y=87037 x-$ $7949.4, R^{2}=0.9992$. The linearity range of the both compounds is within $0.25-1.5 \mu \mathrm{g}$.

\subsection{Degree of Precision}

Apply 7 bands of the CRS solution of jujuboside a and jujuboside b at $2 \mu \mathrm{L}$ per band level and apply 9 bands of $E S Z R, 1.5 \mu \mathrm{L}$ each on silica gel plate respectively. Developing, visualizing, photographing and digital scanning plus integration-calculation as described in 2.2. For ensuring the effective determination, the first step is to select the valid lanes and ignore the contaminated and the bands shape tortured lanes in the image. In this case 5 valid lanes of CRS and 6 valid lanes of Extractive of Suan-Zao-Ren (ESZR) (ignore the contaminated and tortured lanes) was carried out sequentially and integrated via Origin Pro. The RSD of integration peak area of the CRS were 1.61 for jujuboside a, 1.54 for jujuboside b $(\mathrm{n}=5)$; in $E S Z R$, jujuboside a 1.19; jujuboside b $1.31(\mathrm{n}=6)$.

\subsection{Recovery}

Following the method in 2.3, spike jujuboside a $1.3 \mu \mathrm{L}$, jujuboside b $0.8 \mu \mathrm{L}$ by means of Linomat 5 into band of the $\operatorname{ESZR}(0.7 \mu \mathrm{L}$ per band), the measured data as Table 1 .

\section{Testing Results}

\subsection{The Contents of Jujuboside a and Jujuboside b in $S Z R$}

Determined by using the CRS as external standard (jujuboside a $1.049 \%$, jujuboside b $0.619 \%$ ) and by using ESZRR as external standard were carried out respectively. The results in crude drug $S Z R$ and extractive of SZR were shown in Table 2 and Table 3 (Figures 2-4). 


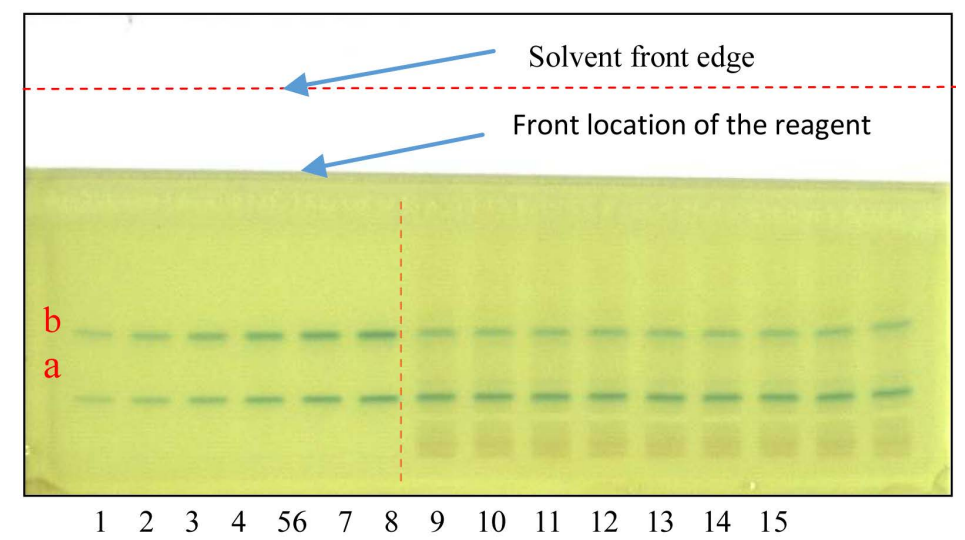

Figure 2. Calibration of jujuboside a and jujuboside b in ESZR. 1 - 6: CRS: jujuboside a and jujuboside b $(1 \mu \mathrm{L}, 1.5 \mu \mathrm{L}, 2 \mu \mathrm{L}, 2.5 \mu \mathrm{L}, 3 \mu \mathrm{L}, 3.5 \mu \mathrm{L}) ; 7$ - 15: ESZR samples, $1.5 \mu \mathrm{L}$ each.

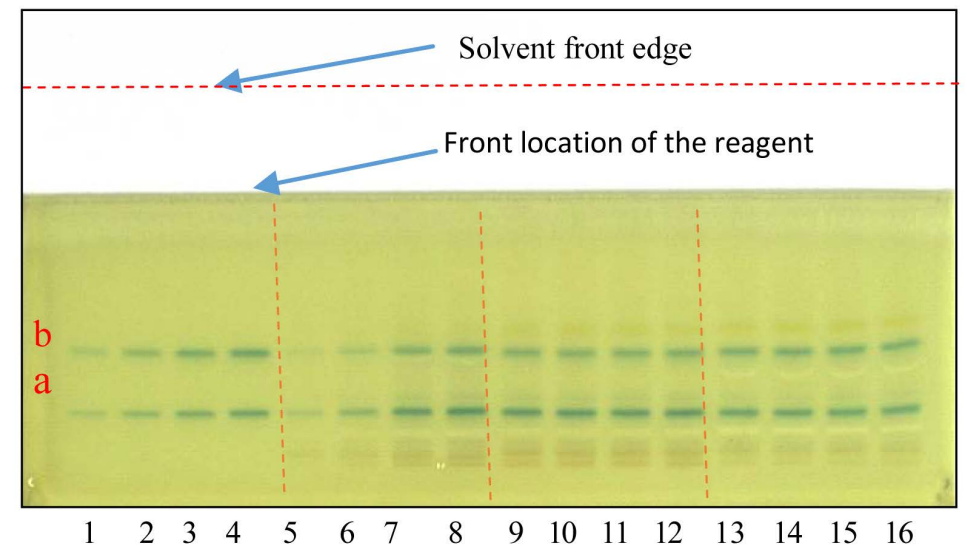

Figure 3. Assay of jujuboside a and jujuboside b in SZR by CRS and ESZRR respectively. 1 - 4: CRS: jujuboside a and jujuboside b $(0.5 \mu \mathrm{L}, 1 \mu \mathrm{L}, 1.5 \mu \mathrm{L}$, $2 \mu \mathrm{L}$ ); 5 - 8: ESZRR, $0.3 \mu \mathrm{L}, 0.6 \mu \mathrm{L}, 1.2 \mu \mathrm{L}, 1.5 \mu \mathrm{L} ; 9$ - 12: crude drug SZR (A), $1.5 \mu \mathrm{L}$; 13 - 16: crude drug SZR (B), $1.5 \mu \mathrm{L}$.

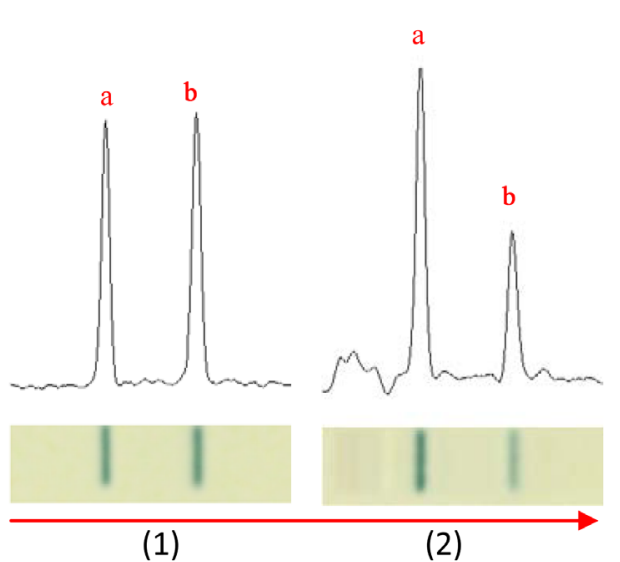

(1)
(2)

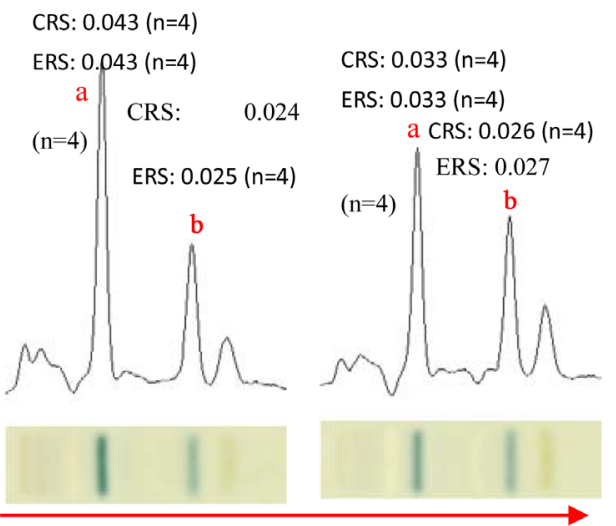

(3)

(4)

Figure 4. HPTLC photographic image of (1) CRS, (2) ESZRR, (3) Crude drug SZR (A), (4) SZR (B) and the corresponding digital scanning profiles. The contents (\%) of jujuboside a and b determined with external standard method by CRS and ERS of SZR (=calibrated extractive SZR). CRS a = jujuboside a; b = jujuboside b. 
Table 2. The contents of jujuboside a and jujuboside b in crude drug SZR (samples A and B).

\begin{tabular}{ccccc}
\hline Content & \multicolumn{2}{c}{ Crude drug SZR $(\mathbf{A})(\mathrm{n}=4)$} & \multicolumn{2}{c}{ Crude drug $\operatorname{SZR}(\mathbf{B})(\mathrm{n}=4)$} \\
\hline & Jujubosidea & Jujuboside b & Jujuboside a & Jujuboside b \\
\hline \% (ERZ) & 0.0433 & 0.0244 & 0.0333 & 0.0259 \\
RSD & 0.0431 & 0.0250 & 0.0332 & 0.0267 \\
\hline
\end{tabular}

CRS (Chemical Reference Substance): jujuboside a, jujuboside b; ESZRR (Extractive Reference Substance) = Extractive Reference Substance of Suan-Zao-Ren.

Table 3. The contents of jujuboside a and jujuboside b in extractive of SZR.

\begin{tabular}{ccc}
\hline content & \multicolumn{2}{c}{$\operatorname{ESZR}(\mathbf{A})(\mathrm{n}=4)$} \\
\hline$\%(\mathrm{CRS})$ & Jujuboside a & Jujuboside b \\
\hline \% (ESZRR) & 1.049 & 0.619 \\
RSD & 1.047 & 0.621 \\
\hline
\end{tabular}

\subsection{The HPTLC Photographic Image and the Digital Scanning Profile}

The HPTLC photographic image and the digital scanning profile were shown in Figures 2-4.

\subsection{Comparison of the Testing Results between Recommended Method Here with the Official HPLC Method}

For evaluating the feasibility of the HPTLC image photo digiscan method, a comparison between HPTLC image digiscan method and official HPLC method was also carried out [6]. It is shown that the recommended method is compatible with the official HPLC method (Table 4).

\section{Discussion}

1) The pilot experimental results showed that quantitative determination through digital scanning the photo of the HPTLC image via software reaches the expected effect which was validated by methodology (Linearity, precision, Recovery) (2.3.1) and the comparison with official HPLC-ELSD method in Chinese Pharmacopoeia.

2) Jujuboside a and jujuboside b reacted with vanillin/sulphuric acid reagent appeared characteristic azure color bands on the light greenish-yellow background of the silica gel plate (Figure 2 and Figure 3). The chromatographic procedure must be carried out in lower relative humidity $(<50 \%)$ and temperature $\left(<25^{\circ} \mathrm{C}\right)$ environment of the lab to keep the air dry and cool. Otherwise no azure color produced. The color of the bands are not stable, it will be faded quickly. So taking the image's photo for quantitation (as well as identification) by digital scanning and measuring the data can be done at anywhere and anytime in convenience and functionality. The qualified HPTLC image is absolutely the prerequisite for conducting this method.

3) As external standard, in spite of the Chemical Reference Substance (CRS), we used the Extractive Reference Substance (ERS) calibrated with CRS (jujuboside a and jujuboside b reference substances) as alternative

Table 4. The contents of jujuboside a and jujuboside b incrude drug SZR by HPLC (ChP 2010) and by HPTLC image photo digiscan (cf. 5.2).

\begin{tabular}{cccc}
\hline Content & Digiscan $n=4$ & HPLC-ELSD $n=4$ & RSD \\
\hline Jujuboside a (\%) & 0.043 & 0.045 & 3.50 \\
Jujuboside b (\%) & 0.025 & 0.024 & 2.32
\end{tabular}

S1: jujuboside a; S2: jujuboside b. 
approach to determining the target marker compounds, the acceptable result showed using the calibrated ERS as standard substance to carry out quantitative analysis would be more simple and low cost [8].

4) The software package ("digiscan" for scanning the photo, "Origin Pro" for integration and "MS Excel” for calculation) were used at the pilot stage, the one-stop integrating software is in progress.

\section{Conclusion}

The photograph of the HPTLC image of herbal medicine as the permanent archive contains the visual qualityrelated data; it can be used as the substitute of HPTLC image plate for quantitative analysis, i.e., digitally scanning the image photo by using software as the virtual scanner replaced the instrumental TLC scanner. The primary results of determination of saponin (jujuboside a and b) in Suan-Zao-Ren (Ziziphi Spinosae Semen) brought out the optimistic foreground. Such a rapid, inexpensive, flexible and feasible method will be expectedly welcomed by the herbal drug industry and sales markets for in-process quality control and market supervising.

\section{Acknowledgements}

The Author appreciates Franky Du who developed the software "digiscan".

\section{References}

[1] Xiao, P.-G. (2002) Chinese Meteria Medica (Upgraded Version) Vol. II. Beijing Chemical Industry Publishing Co., Beijing, 648.

[2] Ma, J.-J., Liu, P. and Ma, B.P. (2011) The Chemical Constituents of Semen Ziziphi Spinosae and Pharmacological Mechanism of Its Sedative and Hypnotic Effects: Research Advances. International Research Journal of Pharmacy, 38 , 206-211.

[3] Zeng, L., Zhang, R.Y. and Wang, X. (1986) Studies on the Constituents of Chinese Traditional Drug Suan Zao Ren. Acta Botanica Sinica, 28, 517-522. (In Chinese with English Abstract)

[4] Zeng, L., Zhang, R.Y. and Wang, X. (1987) Studies on the Constituents of Ziziphus spinosus Hu. Acta Pharmacologica Sinica, 22, 114-120. (In Chinese with English Abstract)

[5] Li, L.-M., Liao, X., Peng, S.L. and Ding, L.-S. (2005) Chemical Constituents from the Seeds of Ziziphus jujube var. spinosa (Bunge) Hu. Journal of Integrative Plant Biology, 47, 494-498. http://dx.doi.org/10.1111/j.1744-7909.2005.00060.x

[6] Chinese Pharmacopoeia Commission (2010) Pharmacopoeia of People’s Republic of China, Volume I. 343-344.

[7] Chinese Pharmacopoeia Commission (2009) TLC Atlas of Chinese Crude Drugs in Pharmacopoeia of the People's Republic of China Volume I. People’s Medical Publishing House, Beijing.

[8] Xie, P.-S., Ma, S.-C., Tu, P.-F., Wang, Z.-T., Stoeger, E. and Bensky, D. (2013) The Prospect of Application of Extractive Reference Substance of Chinese Herbal Medicines. Chinese Medicine, 4, 4125-4136. http://dx.doi.org/10.4236/cm.2013.44016 
Scientific Research Publishing (SCIRP) is one of the largest Open Access journal publishers. It is currently publishing more than 200 open access, online, peer-reviewed journals covering a wide range of academic disciplines. SCIRP serves the worldwide academic communities and contributes to the progress and application of science with its publication.

Other selected journals from SCIRP are listed as below. Submit your manuscript to us via either submit@scirp.org or Online Submission Portal.
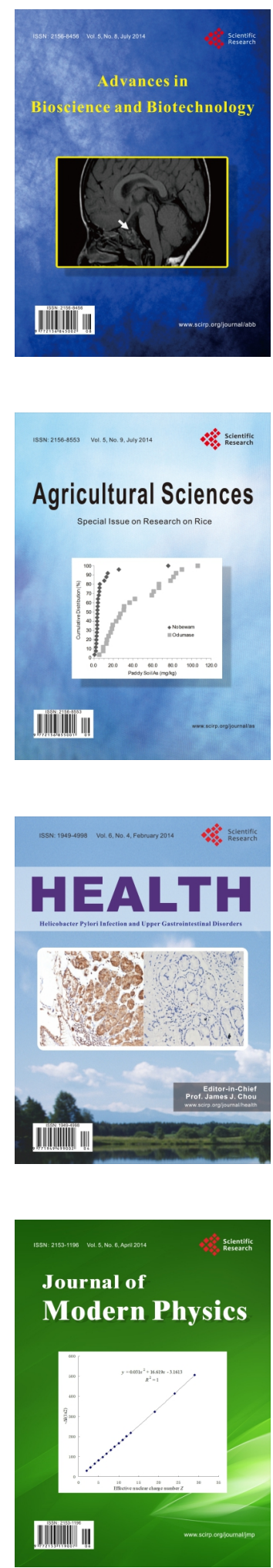
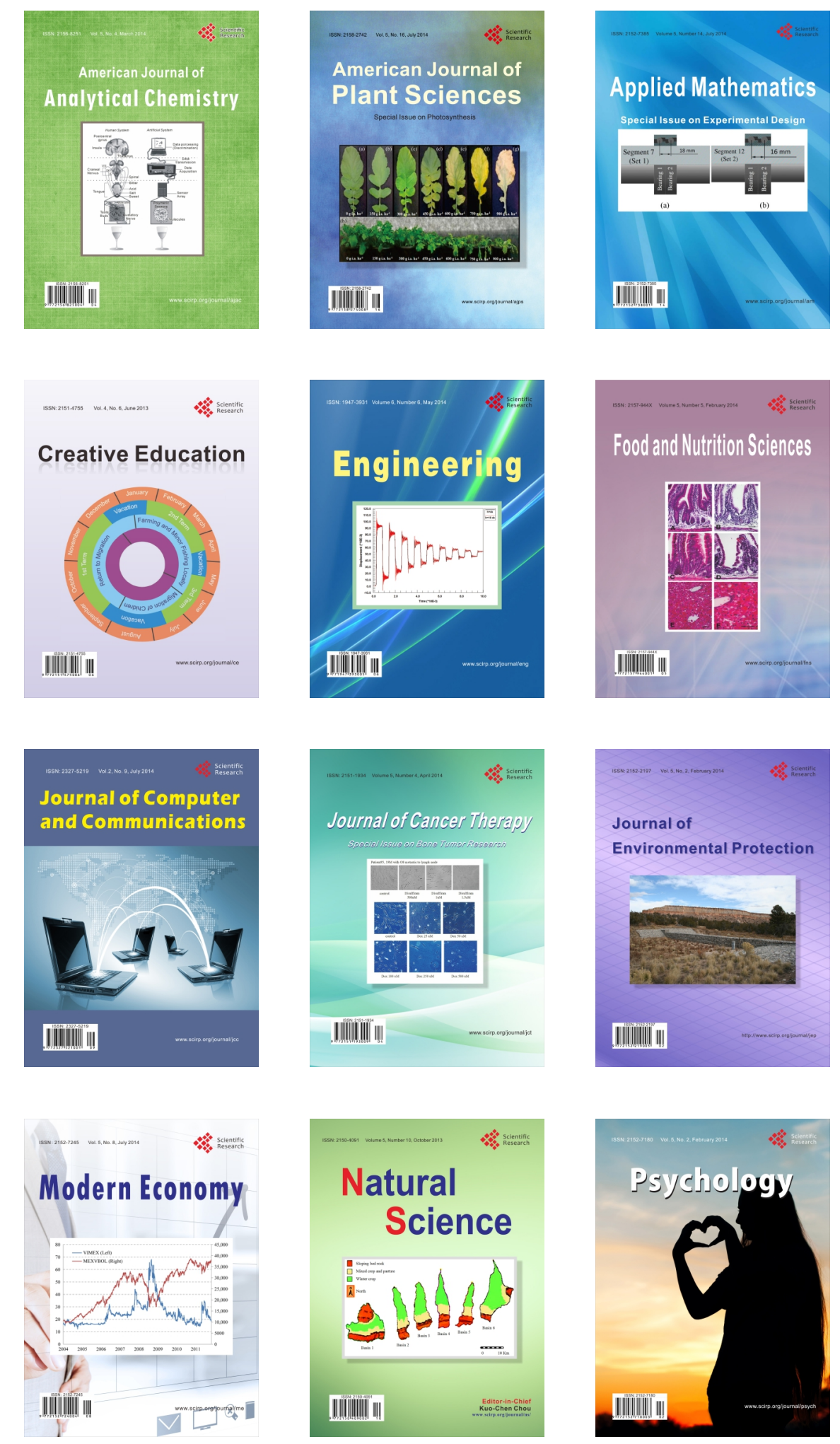